\title{
Classification of Filiform Lie Algebras up to dimension 7 Over Finite Fields
}

\author{
Óscar J. Falcón, Raúl M. Falcón, Juan Núñez, Ana M. Pacheco \\ and M. Trinidad Villar
}

\begin{abstract}
This paper tries to develop a recent research which consists in using Discrete Mathematics as a tool in the study of the problem of the classification of Lie algebras in general, dealing in this case with filiform Lie algebras up to dimension 7 over finite fields. The idea lies in the representation of each Lie algebra by a certain type of graphs. Then, some properties on Graph Theory make easier to classify the algebras. As main results, we find out that there exist, up to isomorphism, six, five and five 6-dimensional filiform Lie algebras and fifteen, eleven and fifteen 7 -dimensional ones, respectively, over $\mathbb{Z} / p \mathbb{Z}$, for $p=2,3,5$. In any case, the main interest of the paper is not the computations itself but both to provide new strategies to find out properties of Lie algebras and to exemplify a suitable technique to be used in classifications for larger dimensions.
\end{abstract}

\section{Introduction}

At present, the study of Lie algebras in general and filiform ones in particular is very extended due to their many applications in several branches of

Key Words: Graphs as a tool; classification; filiform Lie algebra; finite fields.

2010 Mathematics Subject Classification: 17B60, 05C25, 05C20, 05C90, 68W30, 68R10, $05 \mathrm{C} 85$.

Received: January, 2015.

Revised: March, 2015.

Accepted: April, 2015. 
Physics or Engineering. For instance, solvable Lie algebras are used to describe efficiently the scalar field sector of supergravity in relation with its nonperturbative structure encoded in a particular duality group. In this sense, Frè and other authors [8] used solvable Lie algebras as a decisive and useful tool for studying some problems relative to black holes and giving an alternative description of the scalar manifold in a broad class of supergravity theories.

However, the classification of all the types of Lie algebras, except simple and semi-simple ones, is still an unsolved problem. Regarding this topic, in 2005, Schneider [12] got by computational methods 36; 34, and 34 nilpotent 6 -dimensional Lie algebras over the fields $\mathbb{Z} / 2 \mathbb{Z}, \mathbb{Z} / 3 \mathbb{Z}$ and $\mathbb{Z} / 5 \mathbb{Z}$, respectively. All these algebras are classified according to their type. In particular, Schneider got $6 ; 5$, and 5 nilpotent 6 -dimensional Lie algebras of type $[2,1,1,1,1]$ over the fields $\mathbb{Z} / 2 \mathbb{Z}, \mathbb{Z} / 3 \mathbb{Z}$ and $\mathbb{Z} / 5 \mathbb{Z}$, respectively, which correspond to 6 dimensional filiform Lie algebras. In the current paper we confirm these results by using Gröbner bases and we obtain explicitly the law of these filiform algebras. The same is done for 7-dimensional filiform Lie algebras, for which we confirm the existence of $15 ; 11$ and 15 seven-dimensional filiform Lie algebras over those fields. Later, Cical, de Graaf and Schneider also dealt with six-dimensional nilpotent Lie algebras over any field, in 2012 [5].

The main goal of this paper is to step forward in these classifications in the case of filiform Lie algebras over finite fields. The motivation to deal with this type of algebras is due to the following two reasons.

In the first place, filiform Lie algebras, which were introduced by M. Vergne in the late 60's of the past century [14], constitute the most structured subset of nilpotent Lie algebras, which allows us to study and classify them easier than the set of nilpotent Lie algebras. In fact, some of the authors of this paper have deeply studied these algebras in earlier papers and they already got the classification of those algebras over real or complex field for dimensions 10,11 and 12 (see [1], [2] and [7], for instance).

Secondly, with respect to Lie algebras over finite fields, we have already got several results related to classifications of a low-dimensional family of Lie algebras that in a certain sense can be considered a precursor of the filiform Lie algebras: the $n$-dimensional family $\mathcal{F}_{p}$ defined over the field $\mathbb{Z} / p \mathbb{Z}$, with $p$ prime, and having a basis $\left\{u_{1}, \ldots, u_{n}\right\}$, such that: if $r, s<n$, then $\left[u_{r}, u_{s}\right]=0$; and $\left[u_{r}, u_{n}\right]$ is a linear combination of some basis elements $u_{1}, \ldots, u_{n-1}$ with coefficients over the field $\mathbb{Z} / p \mathbb{Z}$ (note that $u_{n}$ does not appear in this last linear combination).

To get those results we develop in this paper a novel research which consists in using Discrete Mathematics as a tool to classify these families. In fact, 
this research was begun in previous papers as $[3,4,10,11]$, for instance. In any case, it is convenient to emphasize that the main interest of our work is not the computations itself. What we try to get is, for one hand, to provide new strategies to find out properties of Lie algebras and for the other hand, to exemplify a suitable technique to be used in classifications for larger dimensions.

The structure of the paper is the following: in the next section some preliminaries on Graph Theory and Lie Theory are given. Sections 3 and 4 are devoted to explain the procedure of linking a 6-dimensional filiform Lie algebra over $\mathbb{Z} / p \mathbb{Z}$, with $p=2,3,5$, with bipartite simple graphs. Similarly, Section 5 deals with 7-dimensional filiform Lie algebras.

As a conclusion, we think that this unexpected use of elements of discrete mathematics could be useful to advance on Lie algebras in the same or similar way as described in this paper. Analogously, Lie theory could be used to obtain new properties about these graphs. In future work, our intention is continue with this topic by increasing both the value of $p$ prime and the dimension of the algebras.

\section{Basic concepts}

This section is devoted to recall some concepts on both Lie and Graph Theories. For a more general review of both theories, the reader can consult $[13,9]$, respectively, for instance.

\subsection{Preliminaries on Lie algebras}

A Lie algebra $\mathfrak{g}$ over a field $K$ is a vector space over $K$ endowed with a second inner law, named bracket product, verifying the following three properties

1. Bilinear: $[u+v, w]=[u, w]+[v, w] ;[u, v+w]=[u, v]+[u, w] ;[\alpha u, \beta v]=$ $\alpha \beta[u, v] \forall \alpha, \beta \in K, \forall u, v, w \in \mathfrak{g}$.

2. Anti-commutative: $[v, u]=-[u, v], \forall u, v \in \mathfrak{g}$.

3. Jacobi identity: $[[u, v], w]+[[v, w], u]+[[w, u], v]=0, \forall u, v, w \in \mathfrak{g}$.

Given a $n$-dimensional Lie algebra $\mathfrak{g}$, its derived series is defined by $\mathcal{C}_{1}(\mathfrak{g})=\mathfrak{g}, \mathcal{C}_{2}(\mathfrak{g})=[\mathfrak{g}, \mathfrak{g}], \mathcal{C}_{3}(\mathfrak{g})=\left[\mathcal{C}_{2}(\mathfrak{g}), \mathcal{C}_{2}(\mathfrak{g})\right], \ldots, \mathcal{C}_{k}(\mathfrak{g})=\left[\mathcal{C}_{k-1}(\mathfrak{g}), \mathcal{C}_{k-1}(\mathfrak{g})\right], \ldots$ 
When there exists $m \in \mathbb{N}$ such that $\mathcal{C}_{m}(\mathfrak{g}) \equiv 0$, the Lie algebra $\mathfrak{g}$ is called solvable.

Given a $n$-dimensional Lie algebra $\mathfrak{g}$, its lower central series is defined as $\mathcal{C}^{1}(\mathfrak{g})=\mathfrak{g}, \mathcal{C}^{2}(\mathfrak{g})=[\mathfrak{g}, \mathfrak{g}], \mathcal{C}^{3}(\mathfrak{g})=\left[\mathcal{C}^{2}(\mathfrak{g}), \mathfrak{g}\right], \ldots, \ldots, \mathcal{C}^{k}(\mathfrak{g})=\left[\mathcal{C}^{k-1}(\mathfrak{g}), \mathfrak{g}\right], \ldots$

and $\mathfrak{g}$ is called nilpotent if there exists $m \in \mathbb{N}$ such that $\mathrm{C}^{m}(\mathfrak{g}) \equiv 0$. It is immediate to see that every nilpotent Lie algebra is solvable.

A nilpotent Lie algebra $\mathfrak{g}$ is said to be filiform, if it is verified that

$$
\operatorname{dim} \mathfrak{g}^{2}=n-2 ; \ldots \operatorname{dim} \mathfrak{g}^{k}=n-k ; \ldots \operatorname{dim} \mathfrak{g}^{n}=0,
$$

with $\operatorname{dim} \mathfrak{g}=n$, and $\mathfrak{g}^{k}=\left[\mathfrak{g}, \mathfrak{g}^{k-1}\right], 2 \leq k \leq n$.

A basis $\left\{e_{1}, \ldots, e_{n}\right\}$ of $\mathfrak{g}$ is called an adapted basis if

$$
\begin{gathered}
{\left[e_{1}, e_{2}\right]=0 ;\left[e_{1}, e_{h}\right]=e_{h-1}(h=3, \ldots, n),} \\
{\left[e_{2}, e_{h}\right]=\left[e_{3}, e_{h}\right]=0(h=3, \ldots, n) .}
\end{gathered}
$$

Note that the definition of filiformity assures that every filiform Lie algebra has an adapted basis. It is easy to deduce that, with respect to this basis, it is verified

$$
\mathfrak{g}^{2} \equiv\left\{e_{2}, \ldots, e_{n-1}\right\}, \mathfrak{g}^{3} \equiv\left\{e_{2}, \ldots, e_{n-2}\right\}, \ldots, \mathfrak{g}^{n-1} \equiv\left\{e_{2}\right\}, \mathfrak{g}^{n} \equiv\{0\}
$$

A filiform Lie algebra $\mathfrak{g}$ is said to be model if the only nonzero brackets between the elements of an adapted basis are the following

$$
\left[e_{1}, e_{h}\right]=e_{h-1}(h=3, \ldots, n) .
$$

These brackets are called brackets due to the filiformity of the algebra and they are not usually indicated in the law of the filiform Lie algebra because they are supposed.

Now, to finish the preliminaries on Lie algebras in general, we recall the following definition.

An isomorphism between Lie algebras is a vector space isomorphism $\phi$ such that $\phi([u, v])=[\phi(u), \phi(v)]$ for each pair of vectors $u$ and $v$ in the algebra.

Then, from here on, we will suppose that all the Lie algebras appearing in this paper are filiform and that all bases are adapted. In the same way, the Jacobi identity $[[a, b], c]+[[b, c], a]+[[c, a], b]=0$, associated with vectors $a, b$ and $c$, will be denoted by $J(a, b, c)=0$.

Let us now recall some concepts on Graph Theory. 


\subsection{Graph Theory terminology}

A simple graph $G=(V(G), E(G))$ consists of two finite sets: $V(G)$, the vertex set of the graph, which is a nonempty set of elements called vertices, and $E(G)$, the edge set of the graph, which is a set of 2 -subsets of vertices. The elements of $E(G)$ are called edges. When $E(G)$ is the empty set, the graph is termed null graph. Every edge $e \in E(G)$ is assigned an unordered pair of vertices $\{u, v\},(u \neq v)$ called the end vertices of $e$, and $e$ is said to join $u$ and $v$.

A weighted graph is a simple graph $G$ in which each edge $e$ has been assigned a real number $w(e)$, called the weight of $e$.

When the weight of an edge is equal to 1 , this value will be omitted in the figure of the graph. For the sake of simplicity we will refer to both simple and weighted graph as a graph when no confusion is produced.

Two vertices defining an edge are called adjacent or neighbours. The set of neighbours of a vertex $v$ is denoted by $N(v)$ and, for a simple graph, the degree of $v$ is $\delta(v)=|N(v)|$.

A bipartite graph is a graph whose vertices can be partitioned into two disjoint sets, $V_{1}$ and $V_{2}$ such that every edge joins a vertex in $V_{1}$ to one in $V_{2}$. An example is shown in Figure 1.
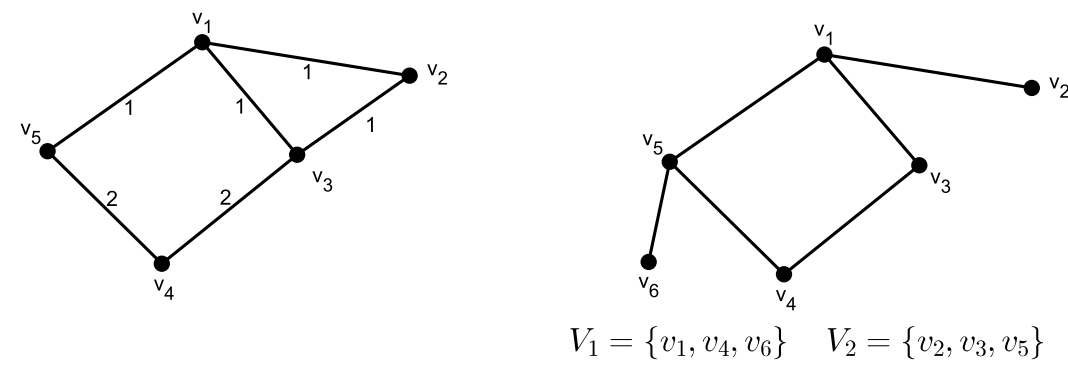

Figure 1: A weighted graph and a bipartite graph.

The concept of adjacency matrix of a graph is very useful in this paper. Let $G$ be a graph with $n$ vertices $v_{1}, v_{2}, \ldots, v_{n}$. The adjacency matrix of $G$, with respect to this particular listing of the vertices of $G$, is the $n \times n$ matrix $M(G)=\left(m_{i j}\right)$ where the $(i, j)-t h$ entry $m_{i j}$ is the weight of the edge joining the vertex $v_{i}$ to the vertex $v_{j}$ is this edge is defined and 0 otherwise.

As an example, $M$ represents the adjacency matrix of the graph in the left of Figure 1. 


$$
M=\left(\begin{array}{lllll}
0 & 1 & 1 & 0 & 1 \\
1 & 0 & 1 & 0 & 0 \\
1 & 1 & 0 & 2 & 0 \\
0 & 0 & 2 & 0 & 2 \\
1 & 0 & 0 & 2 & 0
\end{array}\right)
$$

The adjacency matrix of the weighted graph of Figure 1.

We recall that any bipartite graph $G=(V, E)$ admits an ordering of its vertex set and a partition $V=V_{1} \cup V_{2}$ in such a way that its adjacency matrix can be expressed as follows

$$
M_{G}=\left(\begin{array}{c|c}
\mathcal{O}_{1} & M \\
\hline M^{t} & \mathcal{O}_{2}
\end{array}\right)
$$

where

- $\mathcal{O}_{i}$ denotes the null matrix of dimension $\left|V_{i}\right| \times\left|V_{i}\right|$, for $i=1,2$.

- $M$ is the matrix of dimension $\left|V_{1}\right| \times\left|V_{2}\right|$ collecting the adjacencies between vertices of $V_{1}$ and $V_{2}$.

\section{6-dimensional filiform Lie algebras over $\mathbb{Z} / p \mathbb{Z}$, with $p=$ $2,3,5$}

In the first place, authors would like to explain the reasons for dealing with filiform Lie algebras of dimension 6 . Note that $n=6$ is the lower dimension of a filiform Lie algebra in which non-trivial (non-null or not corresponding to the filiformity) brackets appear. More concretely,

- All the brackets are null in dimension 2. The unique algebra is the abelian.

- For dimension 3 and 4 , all the brackets are either non-null or corresponding to the filiformity. The unique algebra is the model.

- The unique non-trivial bracket of a filiform Lie algebra $\mathfrak{g}$ of dimension 5 is $\left[e_{4}, e_{5}\right]=c_{45}^{2} e_{2}$. Therefore, the corresponding matrix of $\mathfrak{g}$ is $M_{\mathfrak{g}}=\left(c_{45}^{2}\right)$. In a natural way, the adjacency matrix of a bipartite graph $G$ can be constructed as follows $M_{G}=\left(\begin{array}{cc}0 & c_{45}^{2} \\ c_{45}^{2} & 0\end{array}\right)$. The bipartite graphs $G$ 
whose adjacency matrix is $M_{G}$ can be either a pair of non adjacent vertices (for $c_{45}^{2}=0$ ), either a pair of adjacent vertices whose edge has the weight $c_{45}^{2}>0$.

Consequently, we devote this section to classify 6-dimensional filiform Lie algebras over $\mathbb{Z} / p \mathbb{Z}$, with $p=2,3,5$.

Let $\mathcal{F}_{6}^{p}$ be the family of filiform Lie algebras of dimension 6 defined over the finite field $\mathbb{Z} / p \mathbb{Z}$, having a basis $\left\{e_{1}, e_{2}, e_{3}, e_{4}, e_{5}, e_{6}\right\}$. According to the definition of this type of algebras, the law of an algebra $\mathfrak{g} \in \mathcal{F}_{6}^{p}$ with respect to the basis $\left\{e_{k}\right\}_{k=1}^{6}$ is given by the following brackets (filiformity brackets are omitted)

$$
\left[e_{i}, e_{j}\right]=\sum_{h=2}^{4} c_{i j}^{h} e_{h} \quad \text { for } \quad 4 \leq i<j \leq 6
$$

Each algebra $\mathfrak{g} \in \mathcal{F}_{6}^{p}$ is associated with a matrix of order $3 \times 3$ determined by the structure constants of $\mathfrak{g}$, that is

$$
M_{\mathfrak{g}}=\left(\begin{array}{ccc}
c_{45}^{2} & c_{45}^{3} & c_{45}^{4} \\
c_{46}^{2} & c_{46}^{3} & c_{46}^{4} \\
c_{56}^{2} & c_{56}^{3} & c_{56}^{4}
\end{array}\right)
$$

where $c_{45}^{3}=c_{45}^{4}=c_{46}^{4}=0$ due to filiformity reasons (see [2], for instance).

\section{Example 1}

Let $\mathfrak{g} \in \mathcal{F}_{6}^{2}$ be a filiform Lie algebra whose law is given by the following brackets (filiformity brackets have been omitted)

$$
\left[e_{4}, e_{5}\right]=e_{2},\left[e_{4}, e_{6}\right]=e_{3},\left[e_{5}, e_{6}\right]=e_{2}+e_{4} .
$$

The matrix associated with $\mathfrak{g}$ is

$$
M_{\mathfrak{g}}=\left(\begin{array}{ccc}
1 & 0 & 0 \\
0 & 1 & 0 \\
1 & 0 & 1
\end{array}\right)
$$

Next, to classify filiform Lie algebras we study the matrices $M_{\mathfrak{g}}$ and compute the possible values of coefficients $c_{i j}^{k}$ by using Jacobi identities.

Proposition 1. Given $\mathfrak{g} \in \mathcal{F}_{6}^{p}$, the corresponding matrix $M_{\mathfrak{g}}$ is the following, where $a, b, c \in \mathbb{Z} / p \mathbb{Z}$

$$
M_{\mathfrak{g}}=\left(\begin{array}{ccc}
a & 0 & 0 \\
b & a & 0 \\
c & b & a
\end{array}\right)
$$


Proof:

It is straightforward by using $J\left(e_{1}, e_{4}, e_{6}\right)=0$ and $J\left(e_{1}, e_{5}, e_{6}\right)=0$.

Note that this result allows us to set the following

Corollary 1. There exists a bijective map between matrices $M_{\mathfrak{g}}$ and the set of three-dimensional vectors $(a, b, c)$, with $a, b, c \in \mathbb{Z} / p \mathbb{Z}$.

In turn, by denoting the matrix

$$
M_{(a, b, c)}=\left(\begin{array}{ccc}
a & 0 & 0 \\
b & a & 0 \\
c & b & a
\end{array}\right)
$$

to be the corresponding matrix of the vector $(a, b, c)$, Corollary 1 permits us to justify the following relationship between three-dimensional vectors.

Definition 1. The vector $(a, b, c)$ is related with the filiform Lie algebra $\mathfrak{g}$ if the matrix associated with $\mathfrak{g}$ is $M_{(a, b, c)}$.

Two three-dimensional vectors $(a, b, c)$ and $\left(a^{\prime}, b^{\prime}, c^{\prime}\right)$, with components in $\mathbb{Z} / p \mathbb{Z}$, are equivalent (denoted by $\left.(a, b, c) \sim\left(a^{\prime}, b^{\prime}, c^{\prime}\right)\right)$ if their corresponding matrices are associated with isomorphic filiform Lie algebras.

Now, considering these results, 6-dimensional filiform Lie algebras over $\mathbb{Z} / p \mathbb{Z}$ can be classified into equivalence classes according to the field of definition. For computations we have used the symbolic computation package Singular, due to that it is very suitable to deal with Gröbner basis. It is convenient to emphasize that these bases are used to determine whether two vectors are equivalent and could also be used in related works in future. More concretely, we have implemented Algorithm 3.1 to obtain the non-isomorphic 6 -dimensional filiform Lie algebras over $\mathbb{Z} / p \mathbb{Z}$.

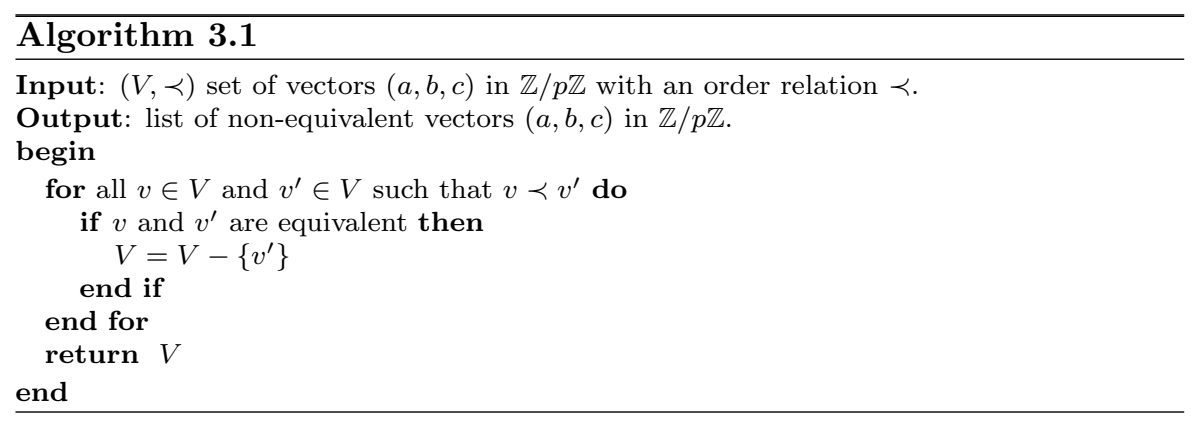

By using Algorithm 3.1 for $p=2,3,5$, the following results were obtained. 
Theorem 1. Up to isomorphism, there exist six 6-dimensional filiform Lie algebras over $\mathbb{Z} / 2 \mathbb{Z}$. They are shown in Table 1 , in which the corresponding vector and the law of each algebra are indicated.

\begin{tabular}{|c|c|c|}
\hline vector & algebra & law \\
\hline$(0,0,0)$ & $\mu_{6,2}^{1}$ & {$\left[e_{4}, e_{5}\right]=0,\left[e_{4}, e_{6}\right]=0,\left[e_{5}, e_{6}\right]=0$} \\
\hline$(0,0,1)$ & $\mu_{6,2}^{2}$ & {$\left[e_{4}, e_{5}\right]=0,\left[e_{4}, e_{6}\right]=0,\left[e_{5}, e_{6}\right]=e_{2}$} \\
\hline$(0,1,0)$ & $\mu_{6,2}^{3}$ & {$\left[e_{4}, e_{5}\right]=0,\left[e_{4}, e_{6}\right]=e_{2},\left[e_{5}, e_{6}\right]=e_{3}$} \\
\hline$(0,1,1)$ & $\mu_{6,2}^{4}$ & {$\left[e_{4}, e_{5}\right]=0,\left[e_{4}, e_{6}\right]=e_{2},\left[e_{5}, e_{6}\right]=e_{2}+e_{3}$} \\
\hline$(1,0,0) \sim(1,0,1)$ & $\mu_{6,2}^{5}$ & {$\left[e_{4}, e_{5}\right]=e_{2},\left[e_{4}, e_{6}\right]=e_{3},\left[e_{5}, e_{6}\right]=e_{4}$} \\
\hline$(1,1,0) \sim(1,1,1)$ & $\mu_{6,2}^{6}$ & {$\left[e_{4}, e_{5}\right]=e_{2},\left[e_{4}, e_{6}\right]=e_{2}+e_{3},\left[e_{5}, e_{6}\right]=e_{3}+e_{4}$} \\
\hline
\end{tabular}

Table 1: 6-dimensional filiform Lie algebras over $\mathbb{Z} / 2 \mathbb{Z}$.

Theorem 2. Up to isomorphism, there exist five 6-dimensional filiform Lie algebras over $\mathbb{Z} / 3 \mathbb{Z}$. Their corresponding representative vectors are

1. $(0,0,0)$.

2. $(0,0, c), \quad c=1,2$.

3. $(0, b, c), \quad b=1,2$ and $c=0,1,2$.

4. $(a, 0, c), \quad a=1,2$ and $c=0,1,2$.

5. $(a, b, c), \quad a, b=1,2$ and $c=0,1,2$.

Theorem 3. Up to isomorphism, there exist five 6-dimensional filiform Lie algebras over $\mathbb{Z} / 5 \mathbb{Z}$. Their corresponding representative vectors are

1. $(0,0,0)$.

2. $(0,0, c), \quad c=1,2,3,4$.

3. $(0, b, c), \quad b=1,2,3,4$ and $c=0,1,2,3,4$.

4. $(a, 0, c), \quad a=1,2,3,4$ and $c=0,1,2,3,4$.

5. $(a, b, c), \quad a, b=1,2,3,4$ and $c=0,1,2,3,4$. 


\section{Associating graphs with 6-dimensional filiform Lie al- gebras over $\mathbb{Z} / p \mathbb{Z}$, with $p=2,3,5$}

Let $\mathfrak{g} \in \mathcal{F}_{6}^{p}$ be an algebra having a basis $\left\{e_{1}, e_{2}, e_{3}, e_{4}, e_{5}, e_{6}\right\}$ and law with respect to this basis is given by the following brackets (filiformity brackets are omitted)

$$
\left[e_{i}, e_{j}\right]=\sum_{h=2}^{4} c_{i j}^{h} e_{h} \quad \text { for } \quad 4 \leq i<j \leq 6
$$

In this section, we show a procedure which links the family $\mathcal{F}_{6}^{p}$ with bipartite weighted graphs.

Let us note that the only non-trivial brackets of $\mathfrak{g}$ are $\left[e_{4}, e_{5}\right],\left[e_{4}, e_{6}\right]$ and $\left[e_{5}, e_{6}\right]$. Moreover, $c_{45}^{3}=c_{45}^{4}=c_{46}^{4}=0$ due to filiformity reasons (see [2], for instance). Consequently, the bracket $\left[e_{4}, e_{5}\right]$ is a linear combination of a unique basis element, $e_{2} ;\left[e_{4}, e_{6}\right]$ is of two basis elements $e_{2}$ and $e_{3} ;$ and $\left[e_{5}, e_{6}\right]$ is of three basis elements $e_{2}, e_{3}$ and $e_{4}$.

This way, each algebra $\mathfrak{g} \in \mathcal{F}_{6}^{p}$ can be represented by a $3 \times 3$ square matrix determined by the structure constants of $\mathfrak{g}$. More concretely, the element in the $k$-th row and $l$-th column is the coefficient of $e_{l+1}$ in the linear combination of the $k$-th non-trivial bracket. For instance, $m_{32}$ is the coefficient of $e_{3}$ in $\left[e_{5}, e_{6}\right]$, as can be checked in $M_{\mathfrak{g}}$.

$$
M_{\mathfrak{g}}=\left(\begin{array}{ccc}
e_{2} & e_{3} & e_{4} \\
c_{45}^{2} & 0 & 0 \\
c_{46}^{2} & c_{46}^{3} & 0 \\
c_{56}^{2} & c_{56}^{3} & c_{56}^{4}
\end{array}\right)\left[\begin{array}{l}
{\left[e_{4}, e_{5}\right]} \\
{\left[e_{4}, e_{6}\right]} \\
{\left[e_{5}, e_{6}\right]}
\end{array}\right.
$$

In a natural way, we construct the adjacency matrix $M_{G}$ of a bipartite graph $G$ as follows

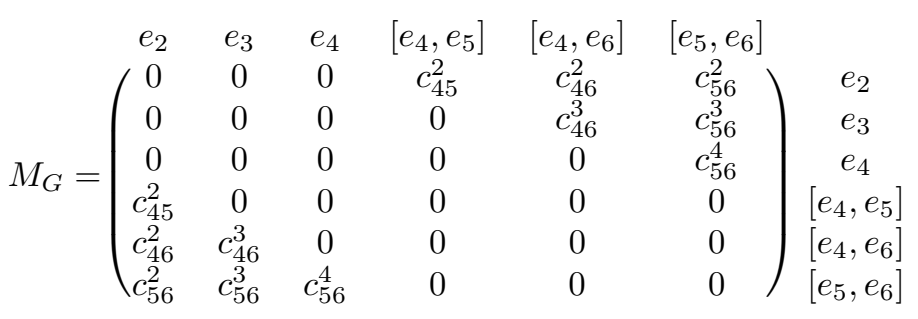


Let us observe that, as it was indicated in section $2.2, M_{G}$ is a symmetric and square matrix of dimension 6 formed by four submatrices of order $3 \times 3$ verifying $M_{G}=\left(\begin{array}{c|c}\mathcal{O} & M_{\mathfrak{g}}^{t} \\ \hline M_{\mathfrak{g}} & \mathcal{O}\end{array}\right)$ where $\mathcal{O}$ denotes the null matrix of dimension 3.

Previous matrix is the adjacency matrix of a bipartite graph $G$ whose set of vertices is partitioned into the subsets $V_{1}=\left\{e_{2}, e_{3}, e_{4}\right\}$ (corresponding with the basis elements which can appear in the brackets of $\mathfrak{g}$ ) and $V_{2}=$ $\left\{\left[e_{4}, e_{5}\right],\left[e_{4}, e_{6}\right],\left[e_{5}, e_{6}\right]\right\}$ (corresponding with the non-trivial brackets of $\mathfrak{g}$ ). Moreover, the weight of the edge joining a vertex $e_{h} \in V_{1}$ and a vertex $\left[e_{i}, e_{j}\right] \in$ $V_{2}$ coincides with $c_{i j}^{h}$, i.e. with the coefficient of $e_{h}$ in $\left[e_{i}, e_{j}\right]$.

In the following figure, we show a representation of a bipartite graph $G$ associated with any algebra $\mathfrak{g} \in \mathcal{F}_{6}^{p}$.

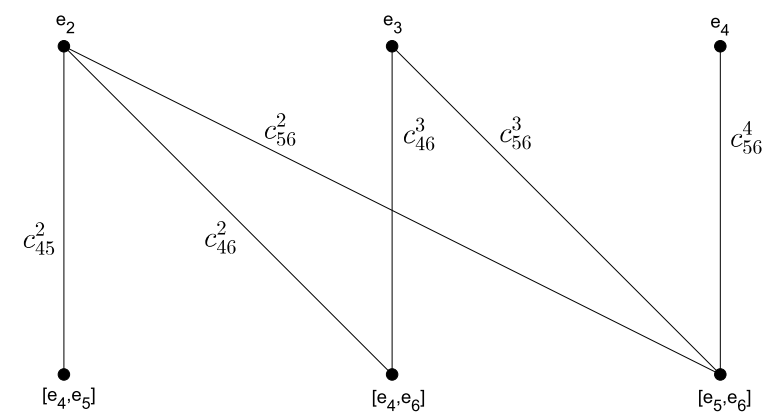

Figure 2: General bipartite graph associated with any algebra of $\mathcal{F}_{6}^{p}$.

As an example of the application of this procedure, let us see the following.

\section{Example 2}

Let now $\mathfrak{g} \in \mathcal{F}_{6}^{3}$ be an algebra whose law is given by

$$
\left[e_{4}, e_{5}\right]=e_{2},\left[e_{4}, e_{6}\right]=e_{2}+e_{3},\left[e_{5}, e_{6}\right]=2 e_{2}+e_{3}+e_{4} .
$$

The matrix representing $\mathfrak{g}$ is

$$
M_{\mathfrak{g}}=\left(\begin{array}{ccc}
1 & 0 & 0 \\
1 & 1 & 0 \\
2 & 1 & 1
\end{array}\right)
$$

In a natural way, we construct the adjacency matrix $M_{G}$ of a bipartite graph $G$ in the following manner 


$$
M_{G}=\left(\begin{array}{cccccc}
e_{2} & e_{3} & e_{4} & {\left[e_{4}, e_{5}\right]} & {\left[e_{4}, e_{6}\right]} & {\left[e_{5}, e_{6}\right]} \\
0 & 0 & 0 & 1 & 1 & 2 \\
0 & 0 & 0 & 0 & 1 & 1 \\
0 & 0 & 0 & 0 & 0 & 1 \\
1 & 0 & 0 & 0 & 0 & 0 \\
1 & 1 & 0 & 0 & 0 & 0 \\
2 & 1 & 1 & 0 & 0 & 0
\end{array}\right) \begin{gathered}
e_{2} \\
e_{3} \\
e_{4} \\
{\left[e_{4}, e_{5}\right]} \\
{\left[e_{4}, e_{6}\right]} \\
{\left[e_{5}, e_{6}\right]}
\end{gathered}
$$

Previous matrix is the adjacency matrix of a bipartite graph $G$ whose set of vertices is partitioned into the subsets $V_{1}=\left\{e_{2}, e_{3}, e_{4}\right\}$ and $V_{2}=$ $\left\{\left[e_{4}, e_{5}\right],\left[e_{4}, e_{6}\right],\left[e_{5}, e_{6}\right]\right\}$. Moreover, the weights of the edges $<e_{2},\left[e_{4}, e_{5}\right]>$, $<e_{3},\left[e_{4}, e_{6}\right]>, \quad<e_{4},\left[e_{5}, e_{6}\right]>, \quad<e_{2},\left[e_{4}, e_{6}\right]>, \quad<e_{3},\left[e_{5}, e_{6}\right]>$ and $<e_{2},\left[e_{5}, e_{6}\right]>$ are $1,1,1,1,1$ and 2 , respectively.

In the following figure, we show a representation of the bipartite graph $G$ associated with the algebra $\mathfrak{g}$.

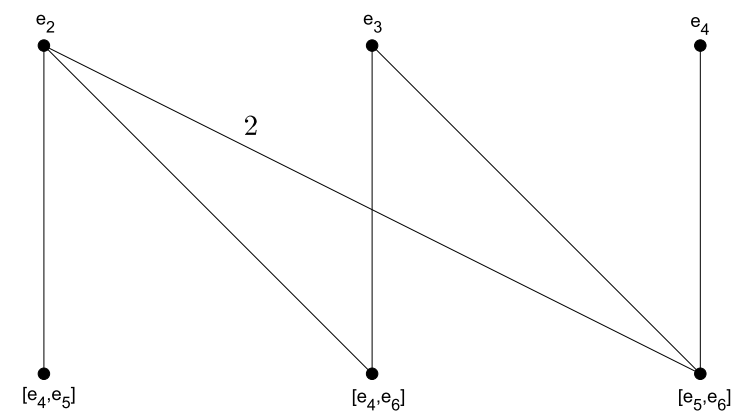

Figure 3: Graph associated with the algebra $\mathfrak{g}$.

Note that with the described procedure, the results given in the above section can be restated by considering the associated graphs with the Lie algebras. One example is the following.

Theorem 4. Up to isomorphism, there exist six 6-dimensional filiform Lie algebras over $\mathbb{Z} / 2 \mathbb{Z}$. They are shown in Figure 4 , in which the corresponding vector, the graph and the law of each algebra are indicated.

As a consequence of previous theorems, every 6-dimensional filiform Lie algebra over $\mathbb{Z} / p \mathbb{Z}$ can be obtained either from its associated vector given 

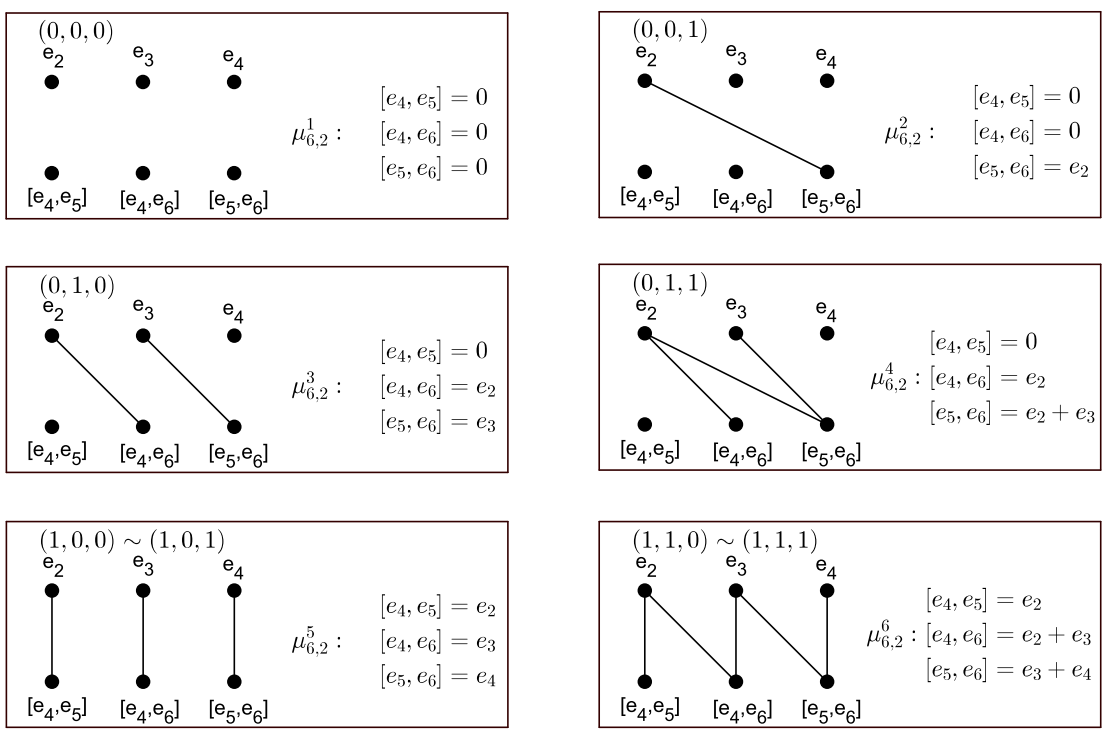

Figure 4: 6-dimensional filiform Lie algebras over $\mathbb{Z} / 2 \mathbb{Z}$.

by Definition 1, either from its representative weighted graph. Moreover, by simple operations over these graphs, all 6-dimensional filiform Lie algebras over $\mathbb{Z} / p \mathbb{Z}$ can be constructed. This can be summarized in the next

Theorem 5. Let $\mathfrak{g} \in \mathcal{F}_{6}^{p}$ be a non-model filiform Lie algebra and let $(a, b, c)$ be its related vector. For $p=2,3,5$, the following statements are held.

1. If $a \neq 0$, the vector $(a+1, b, c)$ (module $p)$ is related with another Lie algebra $\mathfrak{h} \in \mathcal{F}_{6}^{p}$, which is isomorphic with $\mathfrak{g}$, whenever $a+1 \neq 0$ (module $p$ ). The graph associated with $\mathfrak{g}$ has the same weight in edges from vertex $\left[e_{4}, e_{5}\right]$ to $e_{2}$, from $\left[e_{4}, e_{6}\right]$ to $e_{3}$, and from $\left[e_{5}, e_{6}\right]$ to $e_{4}$. Then by increasing the same weight (module p) of edges between each pair of these vertices, a new graph whose associated algebra is isomorphic with $\mathfrak{g}$ is obtained.

2. If $a, b \neq 0$, the vector $(a, b+1, c)$ (module $p$ ) is related with another Lie algebra $\mathfrak{h} \in \mathcal{F}_{6}^{p}$, which is isomorphic with $\mathfrak{g}$, whenever $b+1 \neq 0$ (module $p$ ). The graph associated with $\mathfrak{g}$ has the same weight in edges from vertex $\left[e_{4}, e_{6}\right]$ to $e_{2}$ and from $\left[e_{5}, e_{6}\right]$ to $e_{3}$. Then by increasing the same weight (module $p$ ) of edges between each pair of these vertices, a new graph whose associated algebra is isomorphic with $\mathfrak{g}$ is obtained. 
3. Similarly, if $p=2$ and $a=1$ or else $p \in\{3,5\}$ and whatever $a$ is, it is satisfied $(a, b, c) \sim(a, b, c+1)$, whenever $(a, b, c+1) \neq(0,0,0)$ (module $p$ ). In terms of graphs, it means to increase the weight of an edge (module p) from vertex $\left[e_{5}, e_{6}\right]$ to $e_{2}$ in the graph associated with $\mathfrak{g}$.

Observe that all considered graphs in Theorem 5 are subgraphs of the graph described in Figure 2, where $a=c_{45}^{2}, c_{46}^{3}, c_{56}^{4}, b=c_{46}^{2}, c_{56}^{3}$ and $c=c_{56}^{2}$.

\section{Example 3}

Let us consider the algebra $\mathfrak{g}$ in Example 2 (recall $p=3$ ) whose associated graph is $G$ (shown in Figure 5). Theorem 5 allows us to affirm that

1. The graph $G_{1}$ (shown in Figure 5) is associated with an algebra isomorphic to $\mathfrak{g}$, since $G_{1}$ is obtained from $G$ by increasing one the weight of the edge $<e_{2},\left[e_{5}, e_{6}\right]>$.

2. The graph $G_{2}$ (shown in Figure 5) is associated with an algebra isomorphic to $\mathfrak{g}$, since $G_{2}$ is obtained from $G$ by increasing one the weight of the edges $<e_{2},\left[e_{4}, e_{6}\right]>$ and $<e_{3},\left[e_{5}, e_{6}\right]>$.

3. The graph $G_{3}$ (shown in Figure 5 ) is associated with an algebra isomorphic to $\mathfrak{g}$, since $G_{3}$ is obtained from $G$ by increasing two the weight of the edge $<e_{2},\left[e_{5}, e_{6}\right]>$.
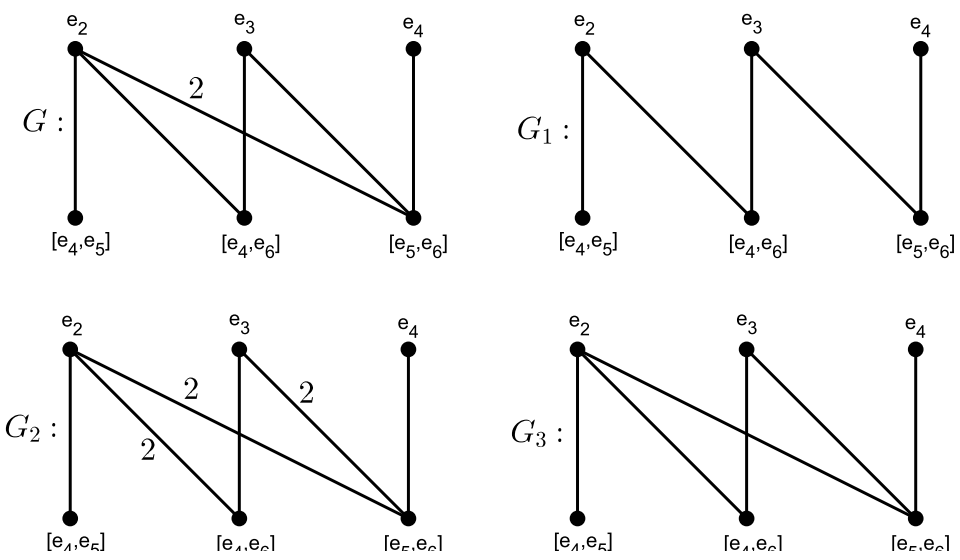

Figure 5: Different graphs associated with isomorphic algebras. 


\section{7-dimensional filiform Lie algebras over $\mathbb{Z} / p \mathbb{Z}$, with $p=$} $2,3,5$

In this section, previous results obtained in dimension 6 are generalized to dimension 7 by using the same procedure. So, we only include the main differences between both dimensions and the final results.

Let $\mathcal{F}_{7}^{p}$ be the family of filiform Lie algebras of dimension 7 defined over the finite field $\mathbb{Z} / p \mathbb{Z}$, having a basis $\left\{e_{1}, e_{2}, e_{3}, e_{4}, e_{5}, e_{6}, e_{7}\right\}$. Each algebra $\mathfrak{g} \in \mathcal{F}_{7}^{p}$ is associated with the matrix of order $4 \times 6$

$$
M_{\mathfrak{g}}=\left(\begin{array}{cccc}
c_{45}^{2} & c_{45}^{3} & c_{45}^{4} & c_{45}^{5} \\
c_{46}^{2} & c_{46}^{3} & c_{46}^{4} & c_{46}^{5} \\
c_{47}^{2} & c_{47}^{3} & c_{47}^{4} & c_{47}^{5} \\
c_{56}^{2} & c_{56}^{3} & c_{56}^{4} & c_{56}^{5} \\
c_{57}^{2} & c_{57}^{3} & c_{57}^{4} & c_{57}^{5} \\
c_{67}^{2} & c_{67}^{3} & c_{67}^{4} & c_{67}^{5}
\end{array}\right)
$$

Due to the filiformity the two first rows and the last column of the previous matrix are null, $M_{\mathfrak{g}}$ can be reduced to the following matrix of order $4 \times 3$

$$
M_{\mathfrak{g}}^{*}=\left(\begin{array}{ccc}
c_{47}^{2} & 0 & 0 \\
c_{56}^{2} & 0 & 0 \\
c_{57}^{2} & c_{57}^{3} & 0 \\
c_{67}^{2} & c_{67}^{3} & c_{67}^{4}
\end{array}\right)
$$

Now, we classify filiform Lie algebras in dimension 7 in the same way as in dimension 6. In the first place, given $\mathfrak{g} \in \mathcal{F}_{7}^{p}$, the corresponding matrix $M_{\mathfrak{g}}$ is the following, where $a, b, c, d \in \mathbb{Z} / p \mathbb{Z}$

$$
M_{\mathfrak{g}}^{*}=\left(\begin{array}{ccc}
a & 0 & 0 \\
b-a & 0 & 0 \\
c & b & 0 \\
d & c & b
\end{array}\right)
$$

We now set that there exists a bijective map between matrices $M_{\mathfrak{g}}^{*}$ and the set of four-dimensional vectors $(a, b, c, d)$, with $a, b, c, d \in \mathbb{Z} / p \mathbb{Z}$.

Then, in a similar way as before, the vector $(a, b, c, d)$ is related with the filiform Lie algebra $\mathfrak{g}$ if the matrix associated with $\mathfrak{g}$ is $M_{(a, b, c, d)}^{*}$. This fact allows us to establish the following relationship between four-dimensional vectors: two four-dimensional vectors $(a, b, c, d)$ and $\left(a^{\prime}, b^{\prime}, c^{\prime}, d^{\prime}\right)$, with components in $\mathbb{Z} / p \mathbb{Z}$, are equivalent (denoted by $\left.(a, b, c, d) \sim\left(a^{\prime}, b^{\prime}, c^{\prime}, d^{\prime}\right)\right)$ if their corresponding matrices are associated with isomorphic filiform Lie algebras. 
In the following figure, we show a representation of a bipartite graph $G$ associated with any algebra $\mathfrak{g} \in \mathcal{F}_{7}^{p}$.

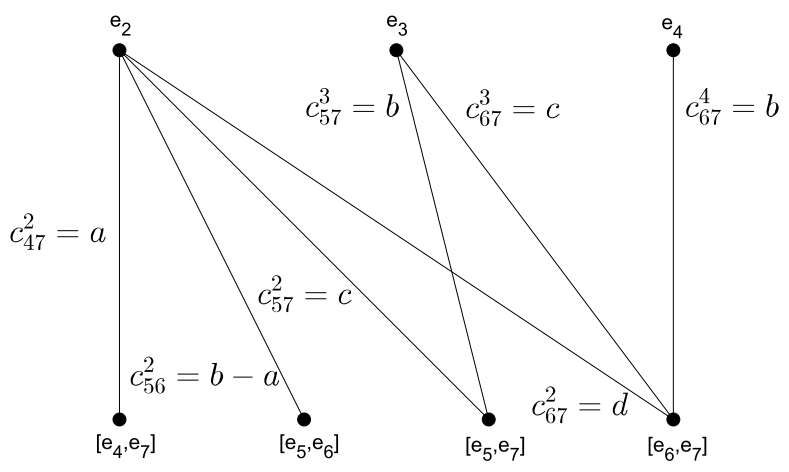

Figure 6: General bipartite graph associated with any algebra of $\mathcal{F}_{7}^{p}$.

Let us note that there exists a significative difference in the case $p=2$. For this reason, we consider this case separately.

\subsection{7-dimensional filiform Lie algebras over $\mathbb{Z} / 2 \mathbb{Z}$}

In this case, a consequence of Engel Theorem for filiform Lie algebras of dimension 7 over a field of characteristic 2 assures the existence of two basis $\mathcal{B}_{1}$ and $\mathcal{B}_{2}$ where, besides of the bracket products of the adapted basis, there exist these other ones [6]:

$$
\begin{aligned}
\text { w.r.t. } \mathcal{B}_{1}: & {\left[e_{4}, e_{6}\right]=e_{2},\left[e_{4}, e_{7}\right]=e_{3},\left[e_{5}, e_{6}\right]=e_{3},\left[e_{5}, e_{7}\right]=e_{2}, } \\
& {\left[e_{6}, e_{7}\right]=e_{3}+c_{67}^{4} e_{4} . } \\
\text { w.r.t. } \mathcal{B}_{2}: & {\left[e_{4}, e_{6}\right]=e_{2},\left[e_{4}, e_{7}\right]=c_{47}^{2} e_{2},\left[e_{5}, e_{6}\right]=e_{3},\left[e_{5}, e_{7}\right]=e_{4}, } \\
& {\left[e_{6}, e_{7}\right]=e_{5} . }
\end{aligned}
$$

As a consequence, the following classification is obtained

Theorem 6. Up to isomorphism, there exist fifteen 7-dimensional filiform Lie algebras over $\mathbb{Z} / 2 \mathbb{Z}$.

- The first eleven are shown in Table 2, in which the corresponding vector and the law of each algebra are indicated.

- Starting from $\mathcal{B}_{1}$, the following two non-isomorphic filiform Lie algebras are obtained 
CLASSIFICATION OF FILIFORM LIE ALGEBRAS UP TO DIMENSION 7 OVER FINITE FIELDS

\begin{tabular}{|c|c|c|}
\hline vector & algebra & law \\
\hline$(0,0,0,0)$ & $\mu_{7,2}^{1}$ & {$\left[e_{4}, e_{7}\right]=0,\left[e_{5}, e_{6}\right]=0,\left[e_{5}, e_{7}\right]=0,\left[e_{6}, e_{7}\right]=0$} \\
\hline$(0,0,0,1)$ & $\mu_{7,2}^{2}$ & {$\left[e_{4}, e_{7}\right]=0,\left[e_{5}, e_{6}\right]=0,\left[e_{5}, e_{7}\right]=0,\left[e_{6}, e_{7}\right]=e_{2}$} \\
\hline$(0,0,1,0)$ & $\mu_{7,2}^{3}$ & {$\left[e_{4}, e_{7}\right]=0,\left[e_{5}, e_{6}\right]=0,\left[e_{5}, e_{7}\right]=e_{2},\left[e_{6}, e_{7}\right]=e_{3}$} \\
\hline$(0,0,1,1)$ & $\mu_{7,2}^{4}$ & {$\left[e_{4}, e_{7}\right]=0,\left[e_{5}, e_{6}\right]=0,\left[e_{5}, e_{7}\right]=e_{2},\left[e_{6}, e_{7}\right]=e_{2}+e_{3}$} \\
\hline$(0,1,0,0) \sim(0,1,0,1)$ & $\mu_{7,2}^{5}$ & {$\left[e_{4}, e_{7}\right]=0,\left[e_{5}, e_{6}\right]=e_{2},\left[e_{5}, e_{7}\right]=e_{3},\left[e_{6}, e_{7}\right]=e_{4}$} \\
\hline$(0,1,1,0) \sim(0,1,1,1)$ & $\mu_{7,2}^{6}$ & {$\left[e_{4}, e_{7}\right]=0,\left[e_{5}, e_{6}\right]=e_{2},\left[e_{5}, e_{7}\right]=e_{2}+e_{3},\left[e_{6}, e_{7}\right]=e_{3}+e_{4}$} \\
\hline$(1,0,0,0) \sim(1,0,0,1)$ & $\mu_{7,2}^{7}$ & {$\left[e_{4}, e_{7}\right]=e_{2},\left[e_{5}, e_{6}\right]=e_{2},\left[e_{5}, e_{7}\right]=0,\left[e_{6}, e_{7}\right]=0$} \\
\hline$(1,0,1,0) \sim(1,0,1,1)$ & $\mu_{7,2}^{8}$ & {$\left[e_{4}, e_{7}\right]=e_{2},\left[e_{5}, e_{6}\right]=e_{2},\left[e_{5}, e_{7}\right]=e_{2},\left[e_{6}, e_{7}\right]=e_{3}$} \\
\hline$(1,1,0,0) \sim(1,1,0,1)$ & $\mu_{7,2}^{9}$ & {$\left[e_{4}, e_{7}\right]=e_{2},\left[e_{5}, e_{6}\right]=0,\left[e_{5}, e_{7}\right]=e_{3},\left[e_{6}, e_{7}\right]=e_{4}$} \\
\hline$(1,1,1,0)$ & $\mu_{7,2}^{10}$ & {$\left[e_{4}, e_{7}\right]=e_{2},\left[e_{5}, e_{6}\right]=0,\left[e_{5}, e_{7}\right]=e_{2}+e_{3},\left[e_{6}, e_{7}\right]=e_{3}+e_{4}$} \\
\hline$(1,1,1,1)$ & $\mu_{7,2}^{11}$ & {$\left[e_{4}, e_{7}\right]=e_{2},\left[e_{5}, e_{6}\right]=0,\left[e_{5}, e_{7}\right]=e_{2}+e_{3},\left[e_{6}, e_{7}\right]=e_{2}+e_{3}+e_{4}$} \\
\hline
\end{tabular}

Table 2: 7-dimensional filiform Lie algebras over $\mathbb{Z} / 2 \mathbb{Z}$.

$\mu_{7,2}^{12} \equiv\left[e_{4}, e_{6}\right]=e_{2}, \quad\left[e_{4}, e_{7}\right]=e_{3}, \quad\left[e_{5}, e_{6}\right]=e_{3}, \quad\left[e_{5}, e_{7}\right]=e_{2}$,

$\left[e_{6}, e_{7}\right]=e_{3}$

$\mu_{7,2}^{13} \equiv\left[e_{4}, e_{6}\right]=e_{2}, \quad\left[e_{4}, e_{7}\right]=e_{3}, \quad\left[e_{5}, e_{6}\right]=e_{3}, \quad\left[e_{5}, e_{7}\right]=e_{2}$,

$\left[e_{6}, e_{7}\right]=e_{3}+e_{4}$

- Starting from $\mathcal{B}_{2}$, the following two non-isomorphic filiform Lie algebras are obtained:

$\mu_{7,2}^{14} \equiv\left[e_{4}, e_{6}\right]=e_{2}, \quad\left[e_{4}, e_{7}\right]=0, \quad\left[e_{5}, e_{6}\right]=e_{3}, \quad\left[e_{5}, e_{7}\right]=e_{4}, \quad\left[e_{6}, e_{7}\right]=$ $e_{5}$.

$\mu_{7,2}^{15} \equiv\left[e_{4}, e_{6}\right]=e_{2}, \quad\left[e_{4}, e_{7}\right]=e_{2}, \quad\left[e_{5}, e_{6}\right]=e_{3}, \quad\left[e_{5}, e_{7}\right]=e_{4}$,

$\left[e_{6}, e_{7}\right]=e_{5}$.

\subsection{7-dimensional filiform Lie algebras over $\mathbb{Z} / p \mathbb{Z}$, with $p=3,5$}

Let us note that, in these cases, there does not exist any difference and we only consider the bracket products of the adapted basis. Following the procedure described at the beginning of this section, the following results are obtained.

Theorem 7. Up to isomorphism, there exist eleven 7-dimensional filiform Lie algebras over $\mathbb{Z} / 3 \mathbb{Z}$. For $u, u^{\prime} \in\{1,2\}$ and $v, v^{\prime} \in\{0,1,2\}$, their corresponding representative vectors are

1. $(0,0,0,0)$,

2. $(0,0,0, u)$,

3. $(0,0, u, 0)$, 
4. $\left(0,0, u, u^{\prime}\right)$,

5. $\left(0, u, v, v^{\prime}\right)$,

6. $(u, 0,0, v)$,

7. $\left(u, 0, u^{\prime}, v\right)$,

8. $(u, u, 0,0) \sim\left(u, u, u^{\prime}, 2 u\right)$,

9. $\left(u, u, u^{\prime}, 0\right) \sim(u, u, 0, u)$,

10. $(u, u, v, u) \sim(u, u, v, 2 u)$,

11. $\left(u, 2 u, v, v^{\prime}\right)$.

Theorem 8. Up to isomorphism, there exist thirteen 7-dimensional filiform Lie algebras over $\mathbb{Z} / 5 \mathbb{Z}$. For $u, u^{\prime} \in\{1,2,3,4\}$ and $v, v^{\prime} \in\{0,1,2,3,4\}$, their corresponding representative vectors are

1. $(0,0,0,0)$,

2. $(0,0,0, u)$,

3. $(0,0, u, 0)$,

4. $\left(0,0, u, u^{\prime}\right)$,

5. $\left(0, u, v, v^{\prime}\right)$,

6. $(u, 0,0, v)$,

7. $\left(u, 0, u^{\prime}, v\right)$,

8. $(u, u, v, 0)$,

9. $(u, u, v, u) \sim(u, u, v, 4 u)$,

10. $(u, u, v, 2 u) \sim(u, u, v, 3 u)$,

11. $\left(u, 2 u, v, v^{\prime}\right)$,

12. $\left(u, 3 u, v, v^{\prime}\right)$,

13. $\left(u, 4 u, v, v^{\prime}\right)$.

We can associate graphs with these algebras following the same procedure as in the previous section (we consider that it is not worth to include again the complete details). 


\section{References}

[1] L. Boza Prieto, F.J. Echarte Reula and J. Núñez Valdés, Classification of complex filiform Lie algebras of dimension 10, Algebras, Groups and Geometries 11 (1994), 253-276.

[2] L. Boza Prieto, E. M. Fedriani Martel and J. Núñez Valdés, A new method for classifying complex filiform Lie algebras, Applied Mathematics and Computation 121: 2-3 (2001), 169-175.

[3] L. Boza, E.M. Fedriani y J. Núñez, Una relación entre los pseudo-grafos dirigidos sin aristas repetidas y algunas álgebras de Lie, Actas del IV Encuentro Andaluz de Matemática Discreta (2005), 99-104.

[4] A. Carriazo, L.M. Fernández and J. Núñez, Combinatorial structures associated with Lie algebras of finite dimension, Linear Algebra and its Applications 389 (2004), 43-61.

[5] S. Cicalò, W. de Graaf and C. Schneider, Six-dimensional nilpotent Lie algebras, Linear Algebra Appl. 436:1 (2012), 163-189.

[6] O. J. Falcón, R. M. Falcón and J. Núñez, Isomorphism and isotopism classes of filiform Lie algebras of dimension up to seven, Submitted, 2015.

[7] E. M. Fedriani, Clasificación de las álgebras de Lie filiformes complejas de dimensión 12. Tesis de Licenciatura. Universidad de Sevilla (España), 1997.

[8] P. Frè, V. Gili, F. Gargiulo, A. Sorin, H. Rulik, M. Trigiante, Cosmological backgrounds of superstring theory and solvable algebras: Oxidation and branes, Nucl. Phys. B 685 (2004) 3-64.

[9] F. Harary, Graph Theory, Addison Wesley, Reading, Mass., 1969.

[10] J. Núñez, A. Pacheco y M.T. Villar, Matemática Discreta aplicada al tratamiento de algunos problemas de la Teoría de Lie, Actas de las VI Jornadas de Matemática Discreta y Algorítmica (VIJMDA), (2008) 485492.

[11] J. Núñez, A. Pacheco y M. T. Villar, Study of a family of Lie algebras over $Z / 3 Z$, International Journal of Applied Mathematics and Statistics, Special volume 7:W10 (2010), 40-45.

[12] C. Schneider, A computer-based approach to the classification of nilpotent Lie algebras, Experiment. Math. 14:2 (2005), 153-160. 
CLASSIFICATION OF FILIFORM LIE ALGEBRAS UP TO DIMENSION 7 OVER FINITE FIELDS

[13] V.S. Varadarajan. Lie Groups, Lie Algebras and their Representations. Springer, 1984.

[14] M. Vergne, Cohomologie des algèbres de Lie nilpotentes. Application à l'étude de la variété des algebres de Lie nilpotentes, Bull. Soc. Math. France 98 (1970), 81-116. 